significantly across different specialties (ranging from $90.4 \%$ to $56.5 \%, \mathrm{p}<0.001$ ) and different geographic regions (ranging from $89.9 \%$ to $72.0 \%, \mathrm{p}<0.001)$. Physicians with a higher adherence to treatment guidelines performed significantly more EVT cases per year than those with a lower adherence (median 30, IQR 35 vs. 15, IQR 25, p<0.001). In level 2B scenarios, the overall decision rate in favor of EVT was lower $(66.6 \%)$ and the differences in decision rates between different specialties $(73.7 \%-40 \%, \mathrm{p}<0.001)$ and regions $(73.9 \%$ $47.1 \%, \mathrm{p}<0.001)$ were more pronounced. Again, physicians opting for EVT performed significantly more EVTs per year (median 30, IQR 30 vs. 20, IQR 30, p<0.001).

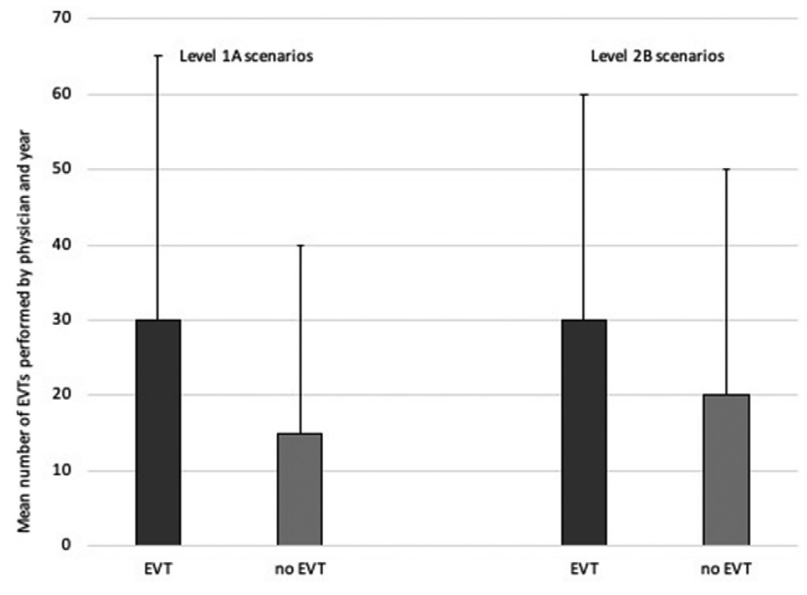

Abstract E-133 Figure 1

Conclusion The high overall adherence to current endovascular stroke treatment guidelines suggests strong consensus within the neurointerventional community. Physician caseload played an important role in our sample.

Disclosures J. Ospel: None. N. Kashani: None. A. Wilson: None. W. Kunz: None. P. Sylaia: None. B. Baxter: None. B. Campbell: None. U. Fischer: None. A. Rabinstein: None. S. Yoshimura: None. J. Heo: None. B. Kim: None. M. Cherian: None. F. Turjman: None. M. Foss: None. B. Menon:None. G. Saposnik: None. M. Hill: None. M. Goyal: None. M. Almekhlafi: 1; C; unrestricted research grant by Stryker to the University of Calgary.

\section{E-134 IN-HOSPITAL COMPLICATIONS OF THROMBOLYTIC TREATMENT FOR ACUTE ISCHEMIC STROKE IN DIALYSIS-DEPENDENT PATIENTS}

K Tong*, A Wang-Selfridge, T Colburn, P Brauer. Anatomy, Kansas City University of Medicine and Biosciences, Kansas City, MO

\subsection{6/neurintsurg-2019-SNIS.209}

Background Intravenous thrombolysis remains an underutilized treatment for acute ischemic stroke (AIS) due to several relative and absolute contraindications. Previous studies have investigated outcomes for the majority of AIS patients receiving thrombolysis without including the subset of renal failure patients requiring dialysis. This study aims to investigate short-term outcomes of thrombolytic treatment for dialysis-dependent patients who suffered in-hospital complications.
Methods This retrospective cohort study utilized data from the 2012-2015Q3 Nationwide Inpatient Sample (NIS). ICD-9 codes identified adult patients (ages 18+) who received thrombolytic treatment for AIS while on dialysis for renal failure, and then further isolated patients diagnosed with in-hospital complications. Complications include intracerebral hemorrhage (ICH), pneumonia, urinary tract infection (UTI), sepsis, deep venous thrombosis (DVT), and pulmonary embolism (PE). Data for patients who were missing important clinical identifiers (age, gender, race, mortality) and did not receive IV thrombolysis or dialysis were excluded. Data analyses assessed hospital mortality rate, length of stay (LOS), inpatient charges, and average age of admission.

Results Of the 5,745 encounters with AIS patients treated with thrombolysis while on dialysis for renal failure, 189 dialysis-dependent patients had in-hospital complications. These patients experienced:

- Lower rate of ICH (1.1\% DD vs. $4.6 \%$ no DD, p<0.0001).

- Higher rate of pneumonia (5.4\% DD vs. $2.9 \%$ no DD, $\mathrm{p}<0.0001)$.

- Lower rate of UTI (2.5\% DD vs. $4.2 \%$ no DD, p<0.0001).

- Higher rate of sepsis $(11.4 \%$ DD vs. $1.9 \%$ no DD, $\mathrm{p}<0.0001)$.

- Higher rate of DVT (15.6\% DD vs. 3.2\% no DD, p = 0.004).

- No significant difference in rate of PE (3.3\% DD vs. $3.3 \%$ no DD).

Conclusion This study aims to inform physicians to better manage dialysis-dependent renal failure patients receiving IV thrombolysis for AIS. These patients experience lower rates of ICH and UTI, and higher rates of pneumonia, sepsis, and DVT. These findings suggest that placing clinical focus on pneumonia, sepsis, and/or DVT prevention before administering IV thrombolysis may be critical for improving short-term in-hospital outcomes for AIS. Future research should aim to investigate different thrombolytic agents to determine the optimal choice for dialysis-dependent patients with pneumonia, sepsis, and DVT, as well as delineate differences in approaches to achieve best outcomes for dialysis-dependent renal failure patients receiving IV thrombolysis.

Disclosures K. Tong: None. A. Wang-Selfridge: None. T. Colburn: None. P. Brauer: None.

\section{E-135 MECHANICAL THROMBECTOMY FOR SMALL AND MEDIUM VESSEL OCCLUSION: A RURAL EXPERIENCE}

${ }^{1} \mathrm{~N}$ Hopkins, ${ }^{1} \mathrm{~V}$ Miller ${ }^{*},{ }^{2} \mathrm{M}$ Manchak, ${ }^{2} \mathrm{~A}$ Drofa, ${ }^{2} \mathrm{G}$ Sachdeva, ${ }^{2} \mathrm{E}$ Kouznetsov. ${ }^{1}$ University of North Dakota, Grand Forks, ND; ${ }^{2}$ Sanford Health, Fargo, ND

\subsection{6/neurintsurg-2019-SNIS.210}

Recent clinical trials have established that treatment of acute ischemic stroke secondary to large vessel occlusion with mechanical thrombectomy leads to improved revascularization and functional outcomes. Thus, the guidelines in treatment of ischemic stroke have been updated to reflect the results found in current literature. However, thrombectomy of small and medium vessel occlusions are a more controversial topic. The aim of the present study is to evaluate the outcomes of patients treated with mechanical thrombectomy for acute ischemic stroke secondary to more distal vessel occlusion and to highlight process times and 